\title{
Doppler Ultrasound in Prediction of the Early Mortality Risk Factors on the Waiting List for Pediatric Liver Transplantation Recipients
}

\author{
T.L. Huang, C.L. Chen, T.Y. Chen, H.H. Weng, T.Y. Lee, Y.S. Chen, Y.C. Chiang, H.L. Eng, C.C. Wang, \\ C.L. Lin, S.H. Wang, H.K. Cheung, B. Jawan, V.H. de Villa, and Y.F. Cheng
}

$\mathrm{D}$ UE TO SHORTAGE of organ donation, limited manpower, and financial resources, a high mortality rate was found on the waiting list for pediatric liver transplantation. The purpose of this study was to document the variable changes in the hemodynamics of hepatic artery and portal vein by Doppler ultrasound and to assess their significance to the mortality in the waiting list and the need for early surgical intervention of liver transplantation for life-saving purposes.

\section{MATERIALS AND METHODS}

There were 36 pediatric patients included in this study (17 girls and 19 boys, age from 0.5 to 7.8 years old, mean age of 1.8 years) with underlying diseases of biliary atresia (29), glycogen storage disease (3), idiopathic portal hypertension (2), type I choledochal cyst (1), and liver hemangioendothelioma (1). We carried out monthly Doppler sonography examination on these potential recipients waiting for a liver transplant for the past 2 years to obtain progressive update data. Ultrasound examinations were performed by a single observer experienced in abdominal and Doppler techniques, using an Acuson 128XP10 (Acuson, Mountain View, Calif, USA) machine with a 3.5- to $7.0-\mathrm{MHz}$ transducer available for color Doppler imaging.

All the measurements of the portal veins were obtained at the extrahepatic portion of the main portal vein near the bifurcation. The portal flow direction was recorded as hepatopetal or hepatofugal. The angle-corrected flow velocity and cross-sectional area of the portal vein were obtained at the same site. The sample volume size for the Doppler scan was set at 1 to $3 \mathrm{~mm}$, about half the diameter of the measured portal vein. This velocity was corrected by the angle (within 30 to 60 degrees) between the long axis of the portal vein and the Doppler beam. We calculated the portal flow volume per body weight as described by Kawasaki et $\mathrm{al}^{1}$ The portal blood flow volume (PBFV) was calculated using the formula: $\mathrm{PBFV}=$ portal vein time average velocity $(\mathrm{TAV})(\mathrm{cm} /$ s) $\times$ portal vein cross sectional area $(A)\left(\mathrm{cm}^{2}\right) \times 60 /$ body weight (BW) (kg). Measurements of the hepatic artery including Doppler US waveform, peak systolic velocity (PSV), and resistance index (RI) were obtained along the hepatic hilum. The PSV of the hepatic artery was detected and corrected along the long axis of the color-coded hepatic artery. The angle between the long axis of the color-coded vessel and the Doppler beam was less than 30 degrees. The Doppler sample volume was set about 1 to $2 \mathrm{~mm}$ as half to the measured arterial caliber. The pulse repetition frequency was maintained at the lowest possible shift range to avoid aliasing. An average of three tracing of all the measurements obtained at the same site were used to generate a mean PSV and RI. The hepatic artery resistance index (HARI) was calculated automatically by the computer software of the ultrasound machine by the formula: HARI $=(\mathrm{S}-\mathrm{D}) / \mathrm{S}$, where $\mathrm{S}$ is peak systolic velocity and $\mathrm{D}$ is end diastolic velocity.

We divided the studied cases into two groups respectively by each of variable factors such as age, body weight, fever (septicemia), esophageal varices (E-V) bleeding, severe jaundice, amount of ascites, PBFV, and HARI, which may be significant as high risk factors of mortality.

\section{RESULTS}

Of the 36 children waiting for liver transplantation, all underwent Doppler ultrasound examination of preoperative evaluation for hepatic hemodynamics in the past 1 year. There were six cases of mortality in the 36 cases studied, and overall cumulative mortality rate in the past 1 year was $18 \%$. There were 10 cases that received liver transplantation for life-saving reasons in subsequent time, and they were all alive; these cases were excluded for survival analysis, thus the mortality of the waiting list should be corrected. Therefore the mortality rate was $23 \%$ higher on these 26 cases of nontransplantation.

\section{ANALYSIS OF THE PREDICTIVE RISK FACTORS}

According to the criteria for every variable risk factor, the studied cases were divided into two groups. There were 21 cases with fever (septicemia), 15 cases with E-V bleeding, 8 cases with moderate to marked ascites, 20 cases of severe jaundice with total serum bilirubin over $10 \mathrm{mg} / \mathrm{dL}, 13$ cases of PBFV less than $9 \mathrm{~mL} / \mathrm{min}-\mathrm{kg}$ body weight, and 6 cases with HARI $>1$. All these clinical variable factors were analyzed for the significance during the past 1 year of

From the Department of Diagnostic Radiology (T.L.H., T.Y.C., H.H.W., T.Y.L., Y.F.C.), General Surgery and Liver Transplant Program, Chang Gung University, Kaohsiung, People's Republic of China, and Memorial Hospital (C.L.C., Y.S.C., Y.C.C., H.L.E., C.C.W., C.L.L., S.H.W., H.K.C., B.J., V.H.D.V.), Kaohsiung Medical Center, Taiwan, People's Republic of China.

Address reprint requests to Yu-Fan Cheng, MD, Department of Diagnostic Radiology, Chang Gung Memorial Hospital, Kaohsiung Medical Center, 123, Ta Pei Road, Niao-Sung, Kaohsiung, Taiwan. 
follow-up by the logistic regression model. The results of analysis of the significance are shown. The ages and body weights were not significant $(P=.482)$. The symptom of fever (septicemia) was also not significant. The factor of E-V bleeding was not so significant with $P=.246$. But the presence of marked ascites had borderline significance with $P=.06$. The 13 cases with $\mathrm{PBFV}<10 \mathrm{~mL} / \mathrm{min}-\mathrm{kg}$ were just half of total studied cases. Logistic regression analysis was done with comparison to the group of PBFV $>10 \mathrm{~mL} /$ min-kg with $P=.056$ and also had significance. The six cases of HARI $>1.0$ was also analyzed with comparison to those of HARI $<1$ with evident significance and $P=.016$ $(P<.05)$. We also combined the two major variable factors of PBFV and HARI in the multiple logistic regression model. Two factors, PBFV $(P=.067)$ and HARI $(P=$ $.220)$, were included in the multiple regression model with $P<.3$ as an inclusive critical significant value.

\section{DISCUSSION}

Doppler ultrasound is routinely used to assess the hepatic and portal vasculature in liver diseases. The portal vein patency and flow direction analysis is well established, but the hepatic arterial waveform and resistance are less well established. $^{2-4}$ There are many reports of increase of arterial resistance in renal parenchymal disease. Especially in rejection of renal transplants, the resistance of inflow was high and was expressed as an increase of arterial resistance index, which was significant in prognosis. ${ }^{5}$ According to the report of Henrion et al, liver cirrhosis in children with ischemic hepatitis all presented with arterial reversed end diastolic flow. ${ }^{6}$ Perfusion of the liver becomes critical in these cases while associated with severe portal hypertension. Therefore, in advanced liver cirrhosis with severe portal hypertension, the hepatic arterial flow is also reduced due to increased intrahepatic vascular resistance. Hepatic hypotension with reduction of total liver inflow may cause liver necrosis and liver failure. ${ }^{7-9}$ Secondary infection of the necrotic liver could result in septicemia.,10

According to the survival analysis for all supposed significant risk factors in our study, all the risk factors of sepsis, bleeding, inadequate PBFV $(<10 \mathrm{~mL} / \mathrm{min}-\mathrm{kg})$, and high HARI $(>1.0)$ had definite significance to mortality. Other variables such as age, body weight, ascites, and severe jaundice were not concluded with evident significance. The results were as we speculated that both risk factors of poor PBFV and high HARI were strongly related with the prognosis and clinical deterioration of our cases studied, especially for the four of six cases of mortality, with both low PBFV ( $<10 \mathrm{~mL} / \mathrm{min}-\mathrm{kg})$ and high HARI $(>1.0)$.

Since 1994, 25 pediatric cases of living related liver transplantation (LRLT) have been performed for end-stage liver disease in our center, and we have achieved $100 \%$ survival rate. The results of pediatric liver transplantation have steadily remained the last choice for resolving this problem. However, due to shortage of cadaver organ donation and limitation of manpower and financial resources in
LRLT, there were still many pediatric patients waiting for a liver transplantation.

In this study, the majority of pediatric patients have biliary atresia ( 29 cases). They were 20 cases shown to be at risk of rapid deterioration in general condition. There were 10 cases with high HARI and poor PBFV and admission in intensive care unit, and four cases have had surgical intervention for life-saving reasons. But unfortunately, the other six cases had no organ donation available, and four cases of mortality happened at last. That is, in six cases of mortality, there were four cases with high HARI $(>1.0$, average of 1.02). There were 10 cases including the four cases with high HARI that have received subsequently liver transplantation for life-saving reasons, and all survived successfully. Thus these cases were excluded for survival analysis. The mortality rate on these 26 cases of nontransplantation was corrected and was higher than $23 \%$. But the mortality of the cases with high HARI (four of six) could be higher than $67 \%$. So recognition of such patients is important, and early surgical intervention for liver transplantation may reduce the waiting list mortality.

In conclusion, two major conclusions as we expected are stated in the following:

1. Decrease and reversal of the portal flow is a poor prognosis in the cases with chronic disease due to high risk of lethal esophageal varices bleeding.

2. Increase of hepatic arterial impedance with high resistant index is the worst prognostic factor that is highly associated with decrease of hepatic arterial inflow and the risk of hepatic failure and sepsis. The fact was that the longer the waiting duration, the worse the clinical condition of the patients. Both high HARI ( $>1.0)$ and poor PBFV ( $<10 \mathrm{~mL} / \mathrm{min}-\mathrm{kg})$ were significant with high risk of early mortality. Such patients could no longer wait and required a liver transplantation for life-saving reasons.

\section{REFERENCES}

1. Kawasaki T, Moriyasu F, Kimura T, et al: Radiology 180: 373, 1991

2. Broide E, Farrant P, Reid F, et al: Liver Transplant Surg 3:604, 1997

3. Lafortune M, Marlean D, Breton G, et al: Radiology 151:27, 1984

4. Yang SS, Wu CH, Chen TK, et al: J Gastroenterol Hepatol 10:36, 1995

5. Rifkin MD, Needleman L, Pasto ME, et al: AJR 148:759, 1987

6. Henrion J, Colin L, Schmitz A, et al: J Clin Gastroenterol $16: 35,1993$

7. Tai DI, Changchien CS, Chen CJ, et al: Am J Gastroenterol 91:545, 1996

8. Bosch J, Pizcuta P, Feu F, et al: Gastroenterol Clin North Am 21:1, 1992 1996

9. Iwao T, Toyonaga A, Shigemori H, et al: Radiology 200:793,

10. Gorka W, Kagalwalla A, McParland BJ, et al: J Clin Ultrasound 24:287, 1996 